\title{
Electron-electron correlations in a dynamical impurity system with a Fermi edge singularity
}

\author{
I. Snyman* \\ School of Physics, University of the Witwatersrand, PO Box Wits, Johannesburg, South Africa and \\ National Institute for Theoretical Physics, Private Bag X1, 7602 Matieland, South Africa
}

(Dated: December 2012)

\begin{abstract}
We study spatial correlations in the ground state of a one-dimensional electron gas coupled to a dynamic quantum impurity. The system displays a non-trivial many-body effect known as the Fermi edge singularity: transitions between discrete internal states of the impurity have a powerlaw dependence on the internal energies of the impurity states. We present compact formulas for the static current-current correlator and the pair correlation function. These reveal that spatial correlations induced by the impurity decay slowly (as the third inverse power of distance) and have a power-law energy dependence, characteristic of the Fermi edge singularity.
\end{abstract}

PACS numbers: $73.40 . \mathrm{Gk}, 72.10 . \mathrm{Fk}$

\section{INTRODUCTION}

The situation where a localized scatterer, with discreet internal quantum mechanical degrees of freedom, is coupled to an electron gas, is a common occurrence both in bulk condensed matter systems ${ }^{1}$ and in nano-meter scale electronic devices. ${ }^{2-4}$ Many non-trivial features of these systems can be explained with the aid of simple dynamic quantum impurity models.$\frac{5}{5}$ For several such models, nonperturbative results have been obtained and these results have played a major role in our understanding of strongly correlated electron systems.

In this context, a phenomenon known as the Fermi edge singularity ${ }^{6}$ has been very influential. Its essential ingredients are a Fermi gas that is initially in a stationary state and a local scattering potential that is abruptly switched on at a time $t_{0}$. For times larger than $t_{0}$, the Fermi gas is no longer in a stationary state. To see just how far from stationary a typical initial state is, it is useful to consider the overlap between the ground states of the Hamiltonian before and after $t_{0}$. It is found that the overlap is zero, and this is known as the orthogonality catastrophe ${ }^{7}$ Since the initial state is far from stationary, the Fermi sea is severely shaken up; $\stackrel{8}{*}$ the local scattering potential creates a multitude of particle hole excitations.

The original theory ${ }^{9,10}$ was formulated to account for singularities in the photo-emission and -absorption spectra in some metals. For this problem the natural quantities to investigate are impurity transition rates. These where found to have a power-law dependence on the internal energies of the impurity levels. It was subsequently shown that the same physics applies to transport through a barrier containing an impurity ${ }^{11}$ level, or through a quantum dot. ${ }^{12,13}$ In recent work, non-equilibrium set-ups in which the Fermi sphere is replaced by a non-equilibrium distribution has been investigated. ${ }^{14-20}$. Complications to the basic theory, for instance what happens if the impurity induces resonant scattering, was also considered ${ }^{21}$ Apart from impurity transition rates, current and noise, $\stackrel{22}{2}$ as well as the quench dynamics of the electron gas ${ }^{23,24}$ have been investigated.
These studies clearly indicate that the dynamical impurity induces significant correlations in the electron gas. A system that displays the Fermi edge singularity can therefore be expected to possess a non-trivially correlated ground state. However, as far as we know, this has not yet been studied. Perhaps the reason is that some common realizations of the Fermi edge singularity rely on explicitly time dependent Hamiltonians. In this work we consider a realization with a time independent Hamiltonian. This allows us to investigate ground state correlations. Correlations in time, such as current noise, ${ }^{22}$ have already been studied. However, spatial correlations, a topic that is receiving significant attention in other impurity systems, ${ }^{25-27}$ have not yet been addressed. We therefore do so in this work.

We consider a set-up in which a localized impurity interacts with one-dimensional electrons in a single channel with a linear dispersion relation. ${ }^{28}$ An example of the type of impurity we have in mind, is the charge qubit formed when a single electron is trapped in a double quantum dot $\stackrel{29,30}{ }$ Our model can also be realized with a Josephson charge qubit.31 Our main result is a compact formula for the current-current correlator $\langle\delta j(x) \delta j(y)\rangle$ (Eq.4.26), that is valid at large separations. The impurity induces no additional correlations between electrons on the same side of the impurity. For electrons on opposite sides of the impurity, impurity induced correlations decay as $1 /|x-y|^{3}$. Owing to the Fermi-edge singularity, the correlator has a power-law dependence on the internal energies of the impurity. The correlations persist beyond the range of the interaction between the electron gas and the impurity. This is in contrast to the expectation values of single particle observables such as the density $\langle\rho(x)\rangle$, which is unaffected by the impurity for $x$ outside the range of the interaction. Because of the linear dispersion relation, the current-current correlator is essentially equal to the pair correlation function $\langle\rho(x) \rho(y)\rangle$. This insight allows for a simple interpretation of our results. If an electron is detected at $x$ to the left of the impurity, the likelihood of finding another at $y$ to the right of the impurity is larger than in the absence of the impurity by 
an amount $\sim 1 /|x-y|^{3}$ and has a power-law dependence on the internal energies of the impurity, characteristic of the Fermi edge singularity.

We present the work as follows: We define the model by means of its Hamiltonian (Sec. 【A ) Since we are dealing with one dimensional electrons with a linear dispersion relation, it is convenient to bosonize them. In Sec.IIB we collect the relevant bosonization results. We are interested in the model because it displays a Fermi edge singularity. We start Sec.IIC by reviewing how the conventional theory of the Fermi edge singularity applies to the transition rates between internal states of the impurity. We also take the first step towards studying ground state properties by considering the probability to find the impurity in a given internal state, if the system as a whole is in the ground state. These occupation probabilities turn out to play a central role in determining spatial correlations between electrons in the presence of the impurity. We show that the Fermi edge singularity is also manifested in the occupation probabilities. As already stated, our main aim is to investigate spatial correlations among the electrons. But before doing so, we need to consider the expectation values of single particle observables. In Sec. III we therefore investigate the average density $\langle\rho(x)\rangle$. We show that the total number of particles displaced by the impurity obeys a generalization of Friedel's sum rule. (A generalized Friedel sum rule has previously been shown to hold for another dynamic impurity system, namely the Anderson impurity model.) Our results for the current-current correlator is presented in Sec.IV] and for the pair correlation function in Sec.V. Sec.VI contains a self-contained summary of our main result and conclusions.

\section{MODEL}

Our model describes a localized impurity with an internal state space spanned by the orthonormal basis $B=\{|\alpha\rangle \mid \alpha=1, \ldots, M\}$. It interacts with electrons in a single channel with a linear dispersion relation $E_{k}=k$. The model is inspired by experimental setups in which elecrons in a coherent conductor are coupled to a charge qubit $(M=2): \underline{17,28}$ Since it does not substantially complicate our analysis, we consider arbitrary $M$.

\section{A. Hamiltonian}

The Hamiltonian for our model is $H=H_{0}+H_{T}$, where

$$
\begin{aligned}
& H_{0}=\sum_{\alpha} H_{\alpha} \otimes|\alpha\rangle\left\langle\alpha\left|, \quad H_{T}=\sum_{\alpha \beta} \gamma_{\alpha \beta}\right| \alpha\right\rangle\langle\beta|, \\
& H_{\alpha}=\int_{-L / 2}^{L / 2} d x \psi^{\dagger}(x)\left[-i \partial_{x}+v_{\alpha}(x)\right] \psi(x)+\varepsilon_{\alpha} .
\end{aligned}
$$

Here $H_{\alpha}$ describes the fermions when the impurity is in state $|\alpha\rangle$ and $\varepsilon_{\alpha}$ is the internal energy of the impurity state $|\alpha\rangle$. The fermion creation and annihilation operators $\psi^{\dagger}(x)$ and $\psi(x)$ obey periodic boundary conditions on the interval $(-L / 2, L / 2]$. We will eventually send the system size $L$ to infinity. The electrostatic potential $v_{\alpha}(x)$ depends on the state of the impurity. Since our aim in this work is to investigate many-body correlations that persist beyond the range of the interaction between the impurity and the Fermi gas, it is convenient to assume that all the potentials $v_{\alpha}$ have bounded support. We define the scattering region as the shortest interval $l=\left[x_{-}, x_{+}\right]$such that $v_{\alpha}(x)=0$ for all $\alpha$ and all $x \notin l$. The term $H_{T}$ induces tunnelling between impurity states. Without loss of generality, we set $\gamma_{\alpha \alpha}=0$.

The ground state $\left|F_{\alpha}\right\rangle$ of the fermion Hamiltonian $H_{\alpha}$ is a Fermi-sea in which all (the infinitely many) negative energy orbitals associated with the single particle Hamiltonian $-i \partial_{x}+v_{\alpha}(x)$ are occupied, and all positive energy orbitals are empty.

The scattering states of $-i \partial_{x}+v_{\alpha}(x)$ are of the form $\exp \left[i k x-i \int_{-\infty}^{x} d x^{\prime} v_{\alpha}\left(x^{\prime}\right)\right]$. This leads to an identification of

$$
\delta_{\alpha}=-\frac{1}{2} \int_{-\infty}^{\infty} d x v_{\alpha}(x)
$$

as the scattering phase shift associated with the potential $v_{\alpha}(x)$. Phase shifts play an important role in impurity physics.

\section{B. Bosonization}

The infinite Fermi sea represented by a state such as $\left|F_{\alpha}\right\rangle$ must be handled with care. Finite observable quantities are often represented as the differnece between infinite quantities. In such calculations, an uncontrolled rearrangement of terms can lead to incorrect answers $\stackrel{32,33}{ }$ For one-dimensional Fermi systems, such as the one we are studying, a standard and elegant method to treat the infinitely deep Fermi sea correctly, is provided by bosonization 34,35 In fact, one of the pioneering applications of bosonization was to the Fermi edge singularity ${ }^{36}$ Through bosonization, our model is mapped onto a spin-boson model ${ }^{37}$ with a bath that is Ohmic at low energies $\stackrel{28}{=}$ For our purposes the following bosonization results are required. The Fourier components of the density operator

$$
\rho_{q}=\int_{-L / 2}^{L / 2} d x e^{-i q x} \psi^{\dagger}(x) \psi(x),
$$

with $q=2 \pi n / L, n=0, \pm 1, \pm 2, \ldots$ obey bosonic commutation relations

$$
\left[\rho_{q}, \rho_{q^{\prime}}\right]=\frac{L q}{2 \pi} \delta_{q,-q^{\prime}}
$$

The fermion Hamiltonians $H_{\alpha}$ can be expressed in terms of $\rho_{q}$ as $H_{\alpha}=h_{\alpha}+E_{0 \alpha}$ where

$$
h_{\alpha}=\sum_{q>0} \frac{2 \pi}{L}\left(\rho_{-q}+\frac{v_{\alpha,-q}}{2 \pi}\right)\left(\rho_{q}+\frac{v_{\alpha, q}}{2 \pi}\right) .
$$


Here $v_{\alpha, q}=\int_{-L / 2}^{L / 2} d x e^{-i q x} v_{\alpha}(x)$ are the Fourier components of the potentials $v_{\alpha}(x)$ and

$$
E_{0 \alpha}=\varepsilon_{\alpha}+\frac{\rho_{0} v_{\alpha, 0}}{L}-\sum_{q>0} \frac{2 \pi}{L}\left|\frac{v_{\alpha, q}}{2 \pi}\right|^{2},
$$

is the ground state energy of $H_{\alpha}$ as a function of $\rho_{0}$, the number of electrons in the system, up to an $\alpha$ independent constant. We will work with a fixed number of particles. The number operator $\rho_{0}$, and therfore also $E_{0 \alpha}$, can then be treated as real numbers in stead of operators. Note that $E_{0 \alpha}$ can be varied independently of $h_{\alpha}$ by adjusting the internal energy $\varepsilon_{\alpha}$ of the impurity state $\alpha$. We define the energy differences

$$
\omega_{\alpha \beta}=E_{0 \alpha}-E_{0 \beta} .
$$

For $q>0$,

$$
\rho_{q}\left|F_{\alpha}\right\rangle=-\frac{v_{\alpha, q}}{2 \pi}\left|F_{\alpha}\right\rangle,
$$

i.e. the Fermi sea $\left|F_{\alpha}\right\rangle$ is an eigenstate (coherent state) of the bosonic annihilation operator $\rho_{q}$.

\section{Fermi edge singularity}

The Fermi edge singularity manifests as power law singularities in inelastic transition rates between internal states of the impurity, as a function the energy differences $\omega_{\alpha \beta}$. Denote the many-body eigenstates of $H_{\beta}$ by $\left|m_{\beta}\right\rangle$, and let $E_{m \beta}$ be the associated energies. Assuming an initial state $\left|F_{\alpha}\right\rangle \otimes|\alpha\rangle$, the total transition rate $W_{\beta \alpha}\left(\omega_{\beta \alpha}\right)$ to configurations with the impurity in state $|\beta\rangle$, calculated to second order in $H_{T}$ using Fermi's golden rule, is given by

$$
\begin{aligned}
& W_{\beta \alpha}\left(\omega_{\beta \alpha}\right) \\
& =2 \pi\left|\gamma_{\beta \alpha}\right|^{2} \sum_{m} \delta\left(E_{m \beta}-E_{0 \alpha}\right)\left|\left\langle F_{\alpha} \mid m_{\beta}\right\rangle\right|^{2} \\
& =\left|\gamma_{\beta \alpha}\right|^{2} \sum_{m} \int_{-\infty}^{\infty} d t e^{i\left(E_{m \beta}-E_{0 \alpha}\right) t}\left|\left\langle F_{\alpha} \mid m_{\beta}\right\rangle\right|^{2} \\
& =\left|\gamma_{\beta \alpha}\right|^{2} \int_{-\infty}^{\infty} d t e^{i \omega_{\beta \alpha} t}\left\langle F_{\alpha}\left|e^{i h_{\beta} t} e^{-i h_{\alpha} t}\right| F_{\alpha}\right\rangle .
\end{aligned}
$$

The canonical theory of the Fermi edge singularity provides an expression (the so-called closed loop factor) $\underline{10,12}$ for the expectation value $\left\langle F_{\alpha}\left|e^{i h_{\beta} t} e^{-i h_{\alpha} t}\right| F_{\alpha}\right\rangle$. The closed loop factor is analytic in the upper half of the complex plain. For large $|t|$, its asymptotic behaviour is of the form $(i \Lambda t)^{-\Delta_{\alpha \beta}^{2}}$ where

$$
\Delta_{\alpha \beta}=-\left(\delta_{\alpha}-\delta_{\beta}\right) / \pi .
$$

A branch cut in the lower half of the complex $t$ plane is understood, and the branch with $\arg (t)=0$ for real positive $t$ is implied. $\Lambda$ is a large energy scale. In our model, with an infinitely deep Fermi-sea, it is of the order of the inverse of the range of the interaction of the impurity and the Fermi gas. The interpretation is that an interaction with range $\Lambda^{-1}$ can excite particle-hole pairs with energies $\sim \Lambda$. In models where the inverse of the interaction range is larger than the Fermi energy, measured from the bottom of the Fermi sea, $\Lambda$ is of the order of the Fermi energy. From the asymptotics and analiticity structure of the closed loop factor, the asymptotic behaviour of the rates $W_{\beta \alpha}$ can be extracted at small energy differences $\omega_{\beta \alpha}$.

$$
W_{\alpha \beta}\left(\omega_{\beta \alpha}\right)=\left.\left|\gamma_{\alpha \beta}\right|^{2} C_{\alpha \beta} \Delta_{\alpha \beta}^{2} \theta(\omega)\left(\frac{\omega}{\Lambda}\right)^{\Delta_{\alpha \beta}^{2}} \frac{1}{\omega}\right|_{\omega=-\omega_{\beta \alpha}} .
$$

$C_{\alpha \beta}$ is a constant that tends to a value of order one when $\Delta_{\alpha \beta} \rightarrow 0$. The result for the rate breaks down at energies $\omega_{\alpha \beta}$ corresponding to excitations with wave-lengths $\omega_{\alpha \beta}^{-1}$ short enough to resolve the spatial structure of the interaction potentials $v_{\alpha}(x)$ and $v_{\beta}(x)$. For energies larger than $\Lambda$, the transition rate becomes exponentially suppressed. There is also another constraint. For perturbation theory in $\gamma_{\alpha \beta}$ to be valid, the ratio $W_{\beta \alpha}\left(\omega_{\beta \alpha}\right) / \omega_{\alpha \beta}$ must be much smaller than one $\stackrel{38}{=}$ For $\Delta_{\alpha \beta}^{2}<2$ this imposes the constraint $\frac{39}{}$ that

$$
\frac{\omega_{\alpha \beta}}{\Lambda} \gg\left(\frac{C_{\alpha \beta} \Delta_{\alpha \beta}^{2}\left|\gamma_{\alpha \beta}\right|^{2}}{\Lambda^{2}}\right)^{\frac{1}{2-\Delta_{\alpha \beta}^{2}}} .
$$

The point $\omega_{\beta \alpha}=0$ is known as the Fermi edge threshold. More information regarding the above statements can be found in Ref.28, where we analysed transition rates for essentially the same model in detail. The fact that, for $\Delta_{\beta \alpha}<1$, the transition rate $W_{\omega_{\beta \alpha}}$ becomes large close to the threshold, while for $\Delta_{\beta \alpha}>1$ it becomes small close to the threshold, is an instance of what is known as a Schmidt transition $\underline{\underline{38}}$

The transition rate discussed above is a dynamical property of the model. However, the Fermi edge singularity also manifests in ground state properties. Denote the ground state of the full Hamiltonian by $|\mathrm{GS}\rangle$. We define $n_{\alpha}$ as the probability to find the impurity in state $|\alpha\rangle$ when the system is in $|\mathrm{GS}\rangle$

$$
n_{\alpha}=\langle\mathrm{GS}|(|\alpha\rangle\langle\alpha|)| \mathrm{GS}\rangle .
$$

Let $\lambda$ be the index such that $\left|F_{\lambda}\right\rangle \otimes|\lambda\rangle$ is the ground state of $H_{0}$, the Hamiltonian in the absence of impurity tunnelling. It is straight-forward to show that, to second order in $H_{T}, n_{\alpha}$ with $\alpha \neq \lambda$, is given by

$$
n_{\alpha}=\int_{-\infty}^{0} \frac{d \omega}{2 \pi} \frac{W_{\alpha \lambda}(\omega)}{\left(\omega_{\alpha \lambda}-\omega\right)^{2}} .
$$

(See Appendix $\AA$ for a derivation.) Note that, since $\lambda$ refers to the ground state of $H_{0}, \omega_{\alpha \lambda}$ is positive and the denominator of the integrand is therefore always nonzero. Using the expression of Eq.2.11 for $W_{\alpha \lambda}$ one finds

$$
n_{\alpha}\left(\omega_{\alpha \lambda}\right)=\left|\gamma_{\alpha \lambda}\right|^{2} D_{\alpha \lambda}^{2}\left(\frac{\omega_{\alpha \lambda}}{\Lambda}\right)^{\Delta_{\alpha \lambda}^{2}} \frac{1}{\omega_{\alpha \lambda}^{2}} .
$$


Here $D_{\alpha \lambda}$ is a constant of order one (at least for $\Delta_{\alpha \lambda}^{2}<$ $2)$. The regime of validity of the above equation is the same as that of Eq.2.11 for $W_{\alpha \lambda}$. In particular, for $\Delta_{\alpha \lambda}^{2}<2$, it becomes invalid at sufficiently small $\omega_{\alpha \lambda}$ due to a break-down in perturbation theory in $H_{T}$. For $\Delta_{\alpha \lambda}^{2}>2$ there are additional contributions that are analytic at small $\omega_{\alpha \lambda}$. These contributions are however not important for the quantities that we consider in this work, as they don't give rise to any singular dependence on $\omega_{\alpha \lambda}$. For large $\omega_{\alpha \lambda}$ there is a cross-over from the power law of Eq.2.16 to an inverse square law $n_{\alpha} \sim\left(\omega_{\alpha \lambda}\right)^{-2}$.

\section{CURRENT AND FRIEDEL SUM RULE}

Using the commutation relations between Fourier components of the density operator, the continuity equation $i[\rho(x), H]=\partial_{x} j(x)$ is straight-forwardly derived where the current operator is

$$
j(x)=\rho(x)+\sum_{\alpha} \frac{v_{\alpha}(x)}{2 \pi}|\alpha\rangle\langle\alpha| .
$$

As a preliminary to our goal of studying correlation functions, we consider the average current when the system is in an arbitrary stationary state. Let $D$ be any density matrix that describes a stationary state of the system, i.e. $[H, D]=0$. The expectation value of the commutator of any operator $A$ with the Hamiltonian is zero, i.e.

$$
\langle[A, H]\rangle \equiv \operatorname{tr}\{D[A, H]\}=0 .
$$

From the continuity equation it then follows that $\partial_{x}\langle j(x)\rangle=0$. Integrating from $-\infty$ to $x$ and using the explicit expression of Eq. 3.1 for $j(x)$, we obtain

$$
\langle\rho(x)\rangle=\bar{\rho}-\sum_{\alpha} \frac{v_{\alpha}(x)}{2 \pi} n_{\alpha},
$$

where $n_{\alpha}=\langle\mid \alpha\rangle\langle\alpha \mid\rangle$ is the probability to find the impurity in the state $|\alpha\rangle$ and $\bar{\rho}$ is the density at infinity, which is equal to the homogeneous density in the absence of the impurity. The total charge $\Delta N$ displaced by the impurity, is obtained by subtracting $\bar{\rho}$ and integrating over $x$. In terms of the scattering phase shifts defined in Eq. 2.3. we obtain

$$
\Delta N=\frac{1}{\pi} \sum_{\alpha=1}^{M} n_{\alpha} \delta_{\alpha}
$$

For the case of a static impurity, i.e. $M=1$ and $n_{1}=1$, the result reduces to Friedel's well-known sum rule $\Delta N=\delta / \pi .1$ Eq. 3.4 represents a generalization of Friedel's sum rule to our dynamic impurity model. That such a generalization exists is not unexpected. A classic result in many-body theory is the proof by Langreth 40 that $\Delta N=\delta / \pi$ holds for another dynamic impurity system, namely the Anderson impurity model.
Before considering correlation functions, we define the current-fluctuation operator $\delta j(x)$ through its Fourier transform $\delta j_{q}=\int_{-L / 2}^{L / 2} d x e^{-i q x} \delta j(x)$, where

$$
\begin{aligned}
& \delta j_{q}=\sum_{\alpha} j_{\alpha q}|\alpha\rangle\langle\alpha|-\delta_{q 0} j_{\lambda 0}, \\
& j_{\alpha q}=\rho_{q}+\frac{v_{\alpha q}}{2 \pi}
\end{aligned}
$$

This definition is such that the expectation value of $\delta j_{q}$ with respect to the non-interacting ground state $\left|F_{\lambda}\right\rangle \otimes|\lambda\rangle$ is zero. From the continuity equation follows that the expectation value of $\delta j_{q}$ with respect to the interacting ground state $|\mathrm{GS}\rangle$ is also zero, unless $q=0$. Using the definition of $\Delta_{\alpha \lambda}$ in Eq.2.11 and the fact that $\sum_{\alpha} n_{\alpha}=$ 1 , one furthermore finds

$$
\left\langle\mathrm{GS}\left|\delta j_{q}\right| \mathrm{GS}\right\rangle=\delta_{0 q} \sum_{\alpha} \Delta_{\alpha \lambda} n_{\alpha} .
$$

For a finite interaction strength between the impurity and the Fermi gas, the relative phase shifts $\Delta_{\alpha \lambda}$ are finite, and hence in the thermodynamic limit $L \rightarrow \infty$, the Fourier transformed fluctuations operator $\delta j(x)$ has a zero expectation with respect to the full ground state

$$
\langle\mathrm{GS}|\delta j(x)| \mathrm{GS}\rangle=\left.\frac{1}{L} \sum_{\alpha} \Delta_{\alpha \lambda} n_{\alpha} \rightarrow 0\right|_{L \rightarrow \infty} .
$$

\section{CURRENT-CURRENT CORRELATORS}

The similarity of the generalized Friedel sum rule of $\mathrm{Eq} 3.4$ to the static impurity result, $\Delta N=\delta / \pi$, begs the following question: Given the probabilities $n_{\alpha}$, can the effect of the impurity on the electron gas perhaps be accounted for by a static effective potential $v_{\text {eff }}=$ $\sum_{\alpha} n_{\alpha} v_{\alpha}(x)$ in stead of a dynamic impurity? In this section, we show that the answer is "No". We show this by examening the ground state current-current correlator $\langle\mathrm{GS}|\delta j(x) \delta j(y)| \mathrm{GS}\rangle$, which can in principle be extracted from the statistics of repeated experiments in which the current is measured. (See Appendix [B.)

\section{A. Zero'th order}

We write

$$
\langle\mathrm{GS}|\delta j(x) \delta j(y)| \mathrm{GS}\rangle=c_{0}(x, y)+c_{2}(x, y)+\mathcal{O}\left(\gamma^{4}\right)
$$

where $c_{n}$ is of order of $n$ in $\gamma$. The zero-order term

$$
c_{0}(x, y)=\left\langle F_{\lambda}|\delta j(x) \delta j(y)| F_{\lambda}\right\rangle
$$

becomes singular when $x \rightarrow y$. To deal with this singularity, it is convenient to define a regularised current 
operator

$$
\begin{aligned}
\delta j_{r}(x) & =\frac{1}{L} \sum_{q} e^{i q x} e^{-r|q|} \delta j_{q} \\
& \simeq \int_{-L / 2}^{L / 2} \frac{d x^{\prime}}{\pi} \frac{r}{\left(x-x^{\prime}\right)^{2}+r^{2}} \delta j(x),
\end{aligned}
$$

the last line being valid for $|x| / L \ll 1$. At the end of the calculation the limit $r \rightarrow 0^{+}$is taken and results independent of the regularization is obtained as long as $x \neq y$. From Eqs.2.5 and 2.9 follows that

$$
\left\langle F_{\lambda}\left|\delta j_{p} \delta j_{q}\right| F_{\lambda}\right\rangle=\theta(p) \delta_{p,-q} \frac{L p}{2 \pi}
$$

Performing the inverse Fourier transform we then find

$$
\begin{aligned}
& \left\langle F_{\lambda}|\delta j(x) \delta j(y)| F_{\lambda}\right\rangle \\
& =\frac{-i}{2 \pi L} \partial_{x} \sum_{p>0} e^{i p(x-y+2 i r)} \\
& =\frac{-i}{2 \pi L} \partial_{x}\left[1-e^{i \frac{2 \pi}{L}(x-y+2 i r)}\right]^{-1}
\end{aligned}
$$

Taking the limits $L \rightarrow \infty$ and $r \rightarrow 0^{+}$we find

$$
c_{0}(x, y)=-\frac{1}{(2 \pi)^{2}} \frac{1}{(y-x)^{2}}
$$

This result is independent of $v_{\lambda}(x)$ and represents the exact result for a static impurity.

\section{B. Secdond order}

We obtain the second order correction $c_{2}(x, y)$ to the current-current correlator by expanding $|\mathrm{GS}\rangle$ in Eq.4.1 to second order in $\gamma$. The perturbation expansion of eigenstates can be obtained from a perturbation expansion of the interaction picture time evolution operator describing the situation in which the perturbation is switched on adiabatically [Gell-mann Low theorem]. Thus, to second order in the $\gamma$ 's

$$
\begin{aligned}
|\mathrm{GS}\rangle & =\left|F_{\lambda}\right\rangle \otimes|\lambda\rangle+\sum_{\alpha}\left|\psi_{\alpha}\right\rangle \otimes|\alpha\rangle+\sum_{\alpha_{1} \alpha_{2}}\left|\psi_{\alpha_{1} \alpha_{2}}\right\rangle \otimes\left|\alpha_{2}\right\rangle \\
\left|\psi_{\alpha}\right\rangle & =-i \sum_{\alpha} \gamma_{\alpha \lambda} \int_{-\infty}^{0} d t e^{\eta t} Q_{\alpha \lambda}(t)\left|F_{\lambda}\right\rangle \\
\left|\psi_{\alpha_{1} \alpha_{2}}\right\rangle & =-\sum_{\alpha_{1} \alpha_{2}} \gamma_{\alpha_{2} \alpha_{1}} \gamma_{\alpha_{1} \lambda} \int_{-\infty}^{0} d t_{2} \int_{-\infty}^{t_{2}} d t_{1} e^{\eta\left(t_{1}+t_{2}\right)} Q_{\alpha_{2} \alpha_{1}}\left(t_{2}\right) Q_{\alpha_{1} \lambda}\left(t_{1}\right)\left|F_{\lambda}\right\rangle \otimes\left|\alpha_{2}\right\rangle
\end{aligned}
$$

Here $\eta$ is a small positive constant and the limit $\eta \rightarrow 0^{+}$ must be taken after the expectation value in Eq.4.1 is evaluated. The operators $Q_{\alpha \beta}(t)$ are defined as

$$
Q_{\alpha \beta}(t)=e^{i H_{\alpha} t} e^{-i H_{\beta} t} .
$$

We also define the expectation value

$$
\begin{aligned}
P_{\alpha \beta}(t) & \equiv\left\langle F_{\lambda}\left|Q_{\alpha \beta}(t)\right| F_{\lambda}\right\rangle \\
& =e^{i\left(\omega_{\alpha \lambda}-\omega_{\beta \lambda}\right) t}\left\langle F_{\lambda}\left|e^{i h_{\alpha} t} e^{-i h_{\beta} t}\right| F_{\lambda}\right\rangle .
\end{aligned}
$$

It is convenient to perform a Fourier transform

$$
c_{2}(p, q)=\int_{-L / 2}^{L / 2} d x \int_{-L / 2}^{L / 2} d y e^{-i p x} e^{-i q y} c_{2}(x, y) .
$$

Expanding the ground state $|\mathrm{GS}\rangle$ in $\gamma$ as in Eq.4.7, we obtain

$$
\begin{aligned}
c_{2}(p, q)= & \sum_{\alpha} c_{2}^{\alpha}(p, q) \\
c_{2}^{\alpha}(p, q)= & \left\langle\psi_{\alpha}\left|\delta j_{\alpha p} \delta j_{\alpha q}\right| \psi_{\alpha}\right\rangle+\left\langle F_{\lambda}\left|\delta j_{\lambda p} \delta j_{\lambda q}\right| \psi_{\alpha \lambda}\right\rangle \\
& +\left\langle\psi_{\alpha \lambda}\left|\delta j_{\lambda p} \delta j_{\lambda q}\right| F_{\lambda}\right\rangle .
\end{aligned}
$$

We now substitute $\left|\psi_{\alpha}\right\rangle$ and $\left|\psi_{\alpha \beta}\right\rangle$ from Eqs.4.8 and 4.9 into Eq.4.13. The individual terms in Eq.4.13 are evaluated by exploiting the bosonic commuators $\left[\delta j_{\alpha q}, \delta j_{\beta q^{\prime}}\right]=L q \delta_{q,-q^{\prime}} / 2 \pi$ obeyed by the $j_{\alpha q}$ operators, (cf. Eq.2.5). For $q>0$, the $\delta j_{\alpha q}$ correspond to bosonic annihilation operators, with corresponding creation operators $\delta j_{\alpha q}^{\dagger}=\delta j_{\alpha-q}$. The state $\left|F_{\lambda}\right\rangle$ is a coherent state, i.e. an eigenstate of the annihilation operators, (cf. Eq.2.9)

$$
\delta j_{\alpha q}\left|F_{\lambda}\right\rangle=\Delta_{\alpha \lambda}^{q}\left|F_{\lambda}\right\rangle, \quad \Delta_{\alpha \lambda}^{q}=\frac{v_{\alpha q}-v_{\lambda q}}{2 \pi}
$$

The commutator of $\delta j_{\alpha q}$ with $Q_{\beta \gamma}(t)$ is easily calculated using the $\mathrm{BCH}$ identity. The result is

$$
\left[\delta j_{\alpha q}, Q_{\beta \gamma}\right]=\left(e^{i q t}-1\right) \Delta_{\beta \gamma}^{q} Q_{\beta \gamma}(t)
$$

After some algebra, we obtain the expression $c_{2}^{\alpha}(p, q)=$ $C_{2}^{\alpha}(p, q)+C_{2}^{\alpha}(-p,-q)^{*}$ where 


$$
\begin{aligned}
C_{2}^{\alpha}(p, q)=\lim _{\eta \rightarrow 0^{+}}\left|\gamma_{\alpha \lambda}\right|^{2} \Delta_{\alpha \lambda}^{p} \Delta_{\alpha \lambda}^{q} \int_{-\infty}^{0} d t_{1} \int_{-\infty}^{t_{1}} d t_{2} & \left\{e^{i(p+q) t_{1}}+\theta(p) e^{i q t_{1}}\left[e^{i p t_{2}}-e^{i p t_{1}}\right]+\theta(q) e^{i p t_{1}}\left[e^{i q t_{2}}-e^{i q t_{1}}\right]\right\} \\
& \times e^{\eta\left(t_{1}+t_{2}\right)} P_{\alpha \lambda}\left(t_{2}-t_{1}\right)
\end{aligned}
$$

We change integration variables to $t=t_{1}$ and $\tau=t_{2}-t_{1}$ and perform the $t$ integral to obtain

$$
C_{2}^{\alpha}(p, q)=\lim _{\eta \rightarrow 0^{+}} \frac{\left|\gamma_{\alpha \lambda}\right|^{2}}{2 \eta+i(p+q)} \Delta_{\alpha \lambda}^{p} \Delta_{\alpha \lambda}^{q} \int_{-\infty}^{0} d \tau\left[1+\theta(p)\left(e^{i p \tau}-1\right)+\theta(q)\left(e^{i q \tau}-1\right)\right] e^{\eta \tau} P_{\alpha \lambda}(\tau)
$$

From Eq.4.11 follows that $P_{\alpha \lambda}(t)$ is related to the transition rate $W_{\alpha \lambda}$ by a Fourier transform.

$$
\left|\gamma_{\alpha} \lambda\right|^{2} P_{\alpha \lambda}(t)=\int_{-\infty}^{0} \frac{d \omega}{2 \pi} e^{i\left(\omega_{\alpha \lambda}-\omega\right) t} W_{\alpha \lambda}(\omega) .
$$

Substituting for $\left|\gamma_{\alpha \lambda}\right|^{2} P_{\alpha \lambda}(\tau)$ from Eq.4.18, performing the $\tau$ integral and taking the $\eta \rightarrow 0^{+}$limit, we find

$C_{2}^{\alpha}(p, q)=\frac{\Delta_{\alpha \lambda}^{p} \Delta_{\alpha \lambda}^{q}}{p+q} \int_{-\infty}^{0} \frac{d \omega}{2 \pi} W_{\alpha \lambda}(\omega)\left\{\frac{1}{\omega-\omega_{\alpha \lambda}}+\theta(p)\left[\frac{1}{\omega-\omega_{\alpha \lambda}-p}-\frac{1}{\omega-\omega_{\alpha \lambda}}\right]+\theta(q)\left[\frac{1}{\omega-\omega_{\alpha \lambda}-q}-\frac{1}{\omega-\omega_{\alpha \lambda}}\right]\right\}$,

and hence

$$
c_{2}^{\alpha}(p, q)=\frac{\Delta_{\alpha \lambda}^{p} \Delta_{\alpha \lambda}^{q}}{p+q} \int_{-\infty}^{0} \frac{d \omega}{2 \pi} W_{\alpha \lambda}(\omega)\left\{\operatorname{sign}(p)\left[\frac{1}{\omega-\omega_{\alpha \lambda}-|p|}-\frac{1}{\omega-\omega_{\alpha \lambda}}\right]+\operatorname{sign}(q)\left[\frac{1}{\omega-\omega_{\alpha \lambda}-|q|}-\frac{1}{\omega-\omega_{\alpha \lambda}}\right]\right\} .
$$

Fourier transforming back to $c_{2}^{\alpha}(x, y)$ we obtain

$$
c_{2}^{\alpha}(x, y)=\int_{-\infty}^{0} \frac{d \omega}{2 \pi} W_{\alpha \lambda}(\omega) \int_{-\infty}^{0} d z \int_{0}^{\infty} d z^{\prime}\left[\Delta_{\alpha \lambda}(x+z) \Delta_{\alpha \lambda}\left(y+z^{\prime}\right)+\Delta_{\alpha \lambda}\left(x+z^{\prime}\right) \Delta_{\alpha \lambda}(y+z)\right] h_{\alpha}\left(z^{\prime}-z\right),
$$

where

$$
\Delta_{\alpha \lambda}(x)=\int_{-\infty}^{\infty} \frac{d q}{2 \pi} e^{i q x} \Delta_{\alpha \lambda}^{q}=\frac{v_{\alpha}(x)-v_{\lambda}(x)}{2 \pi},
$$

and

$$
h_{\alpha}(z)=\int_{0}^{\infty} \frac{d q}{\pi} \sin (q z)\left(\frac{1}{\omega_{\alpha \lambda}-\omega}-\frac{1}{\omega_{\alpha \lambda}-\omega+|q|}\right) .
$$

For large $z^{41}$

$$
h_{\alpha}(z) \simeq \frac{2}{\pi} \frac{1}{\left(\omega_{\alpha \lambda}-\omega\right)^{3} z^{3}}=\frac{-1}{\pi} \partial_{\omega_{\alpha \lambda}} \frac{1}{\left(\omega_{\alpha \lambda}-\omega\right)^{2} z^{3}} .
$$

Since $\Delta_{\alpha \lambda}(x)$ is zero for $x \notin l$, i.e. outside the scattering region, the first term in the square brackets in Eq.4.21 gives a non-zero contribution only when $x>x_{-}$ and $y<x_{+}$. The second term in the square brackets on the other hand only gives a non-zero contribution when $x<x_{+}$and $y>x_{-}$. Thus for $x$ and $y$ both to the left $\left(<x_{-}\right)$or both to the right $\left(>x_{+}\right)$of the scattering region, $c_{2}^{\alpha}(x, y)$ is zero. It can be shown that this statement is true to all orders in $H_{T}$.

For $x$ and $y$ on opposite sides of the scattering region, and such that $|x-y| \gg x_{+}-x_{-}$, we can use the large $z$ expansion of $h_{\alpha}(z)$ (cf. Eq.4.24). For $|z| \gg x_{+}-x_{-}$, $h_{\alpha}(z)$ is a slowly varying function on the scale of $x_{+}-x_{-}$. The leading order behavior in $|x-y|$ of $c_{2}^{\alpha}(x, y)$ can be obtained by evaluating $h_{\alpha}\left(z^{\prime}-z\right)$ at $|x-y|$

$$
c_{2}^{\alpha}(x, y) \simeq-\frac{1}{\pi}\left(\Delta_{\alpha \lambda}\right)^{2} \frac{1}{|x-y|^{3}} \partial_{\omega_{\alpha \lambda}} \int_{-\infty}^{0} \frac{d \omega}{2 \pi} \frac{W_{\alpha \lambda}(\omega)}{\left(\omega_{\alpha \lambda}-\omega\right)^{2}},
$$

where $\Delta_{\alpha \lambda} \equiv \int_{-l}^{l} d x \Delta_{\alpha \lambda}(x)$. Substituting into the above the result of Eq.2.15 where the rate $W_{\alpha \lambda}$ was related to the occupation probability $n_{\alpha}$ of impurity level $\alpha$ we obtain the simple final result

$$
c_{2}^{\alpha}(x, y) \simeq-\frac{1}{\pi}\left(\Delta_{\alpha \lambda}\right)^{2} \frac{1}{|x-y|^{3}} \partial_{\omega_{\alpha \lambda}} n_{\alpha}\left(\omega_{\alpha \lambda}\right),
$$

for $\min \{x, y\} \ll x_{-}$and $\max \{x, y\} \gg x_{+}$.

For $\omega_{\alpha \lambda}$ not too large, i.e. not too far from the Fermi edge threshold, the energy dependence of the correlator can be obtained from the expression (Eq.2.16) for $n_{\alpha}$ that we discussed in the section on the Fermi edge singularity. This leads to

$$
c_{2}^{\alpha}(x, y) \sim\left(\omega_{\alpha \lambda}\right)^{\Delta_{\alpha \lambda}^{2}-3} .
$$

For $\Delta_{\alpha \lambda}^{2}<3$ the result diverges close to the Fermi edge threshold. As with the rate $W_{\alpha \lambda}$ and the occupation 
probability $n_{\alpha}$, the divergence signals a breakdown of our expansion in $\gamma$. Understanding this regime is an open problem that we are currently working on.

\section{PAIR-CORRELATION FUNCTION}

Although it can be measured directly (see Appendix B), the current-current correlator that we studied in the previous subsection is a rather abstract quantity. In this subsection we therefore relate it to another correlator, the pair correlation function, that has an appealingly straight forward interpretation. The pair correlation function is defined as

$$
g(x, y)=\left\langle\mathrm{GS}\left|\psi^{\dagger}(y) \psi^{\dagger}(x) \psi(x) \psi(y)\right| \mathrm{GS}\right\rangle .
$$

It measures the likelihood of finding an electron at $x$ together with another electron at $y$. For $x \neq y$ it can also be written as $g(x, y)=\langle\mathrm{GS}|\rho(x) \rho(y)| \mathrm{GS}\rangle$. Since we have $j(x)=\rho(x)$ outside the scattering region (see Eq.3.1), our result for the current-current correlator can be used to obtain a formula for $g(x, y)$ away from the scatterer, namely

$$
g(x, y)=\bar{\rho}^{2}-\frac{1}{(2 \pi)^{2}} \frac{1}{(x-y)^{2}}+\sum_{\alpha} c_{2}^{\alpha}(x, y)+\mathcal{O}\left(\gamma^{4}\right)
$$

The negative sign in front of the second term is due to Fermi statistics: Given that there is an electron at $x$, the likelihood of finding another electron at nearby position $y$ is less than for uncorrelated distinguishable particles. The term $c_{2}(x, y)$, as well as the higher order corrections, are zero if $x$ and $y$ refer to points on the same side of the scatterer, i.e. if $x, y<x_{-}$or $x, y>x_{+}$.

For $\max \{x ; y\} \gg x_{+}$and $\min \{x ; y\} \ll x_{-}$, the approximate result of Eq.4.26 may be used for $c_{2}^{\alpha}$. The sign of $c_{2}^{\alpha}(x, y)$ is determined by $-\left.\partial_{\omega} n_{\alpha}(\omega)\right|_{\omega=\omega_{\alpha \lambda}}$. It is intuitively plausible that the probability $n_{\alpha}$ is a decreasing function of $\omega_{\alpha \lambda}$ : the higher the excitation energy, the less likely it is to find the state $|\alpha\rangle$ occupied. Refering back to Eq. 2.16 in our discussion of the Fermi edge singularity, we see that this is indeed the case not too far from the Fermi edge threshold, provided $\Delta_{\alpha \lambda}^{2}<2$. Exact results for specific model interactions indicate that $n_{\alpha}$ remains a decreasing function of $\omega_{\alpha \lambda}$ also when $\omega_{\alpha \lambda}$ is further away from the threshold or $\Delta_{\alpha \lambda}>2$. This leads to the conclusion that $c_{2}^{\alpha}(x, y)$ is always positive. Thus in the presence of the impurity, the pair correlation function is larger than in the absence of the impurity. This a truly two-particle correlation effect. It cannot be accounted for by an increase in density around the impurity, for the following reasons. The increase in pair correlations occurs outside the scattering region, where $\langle\mathrm{GS}|\rho(x)| \mathrm{GS}\rangle$ is unaffected by the presence of the impurity. Also, the pair correlation function increases regardless of whether the impurity attracts or repel electrons. Finally, the increase in the pair correlation function depends on distance $x-y$ rather than on the distance from $x$ or $y$ to the impurity.

\section{CONCLUSIONS}

We studied a model in which a dynamical quantum impurity is coupled to a degenerate electron gas. The probability $n_{\alpha}$ find the impurity in excited state $|\alpha\rangle$ if the system as a whole is in the ground state, has an energy dependence (cf. Eq.2.16)

$$
n_{\alpha} \sim\left(\omega_{\alpha \lambda}\right)^{\Delta_{\alpha \lambda}^{2}-2}
$$

where $\omega_{\alpha \lambda}$ is the internal energy of impurity state $|\alpha\rangle$ minus the threshold energy of the Fermi edge singularity, and $\Delta_{\alpha \lambda}$ is the relative scattering phase shift that measures the strength of the interaction between impurity state $|\alpha\rangle$ and the Fermi gas. The power-law in Eq.6.1 is due to the Fermi edge singularity, a phenomenon that is usually discussed in the context of impurity transition rates. We note that the divergence of $n_{\alpha}$ at the Fermi edge threshold for $\Delta_{\alpha \lambda}<2$ signals a breakdown in perturbation theory in the tunnelling matrix elements $\gamma_{\alpha \beta}$ of the impurity.

Eq.6.1 establishes that the Fermi edge singularity manifests itself in ground state properties of the impurity. How does it manifest itself in properties of the electron gas? We found that, for any stationary state, the average density of the electron gas is (cf. Eq.3.3)

$$
\langle\rho(x)\rangle=\bar{\rho}-\sum_{\alpha} \frac{v_{\alpha}(x)}{2 \pi} n_{\alpha}
$$

where $\bar{\rho}$ is the homogeneous density in the absence of the impurity and $v_{\alpha}(x)$ is the electrostatic potential that the electron gas is subjected to when the impurity is in state $|\alpha\rangle$. This leads to a generalized Friedel sum rule (cf. Eq.3.4) $\Delta N=\sum_{\alpha} n_{\alpha} \delta_{\alpha} / \pi$ for the number of particles displaced by the impurity, where $\delta_{\alpha}$ is the scattering phase shift associated with $v_{\alpha}(x)$. Two important points about these results are that (1) the effect of the Fermi edge singularity is contained in the occupation probabilities $n_{\alpha}$; and (2) the average density is unaffected by the impurity in regions where the potentails $v_{\alpha}(x)$ are zero, i.e. outside the scattering region.

Having investigated the expectation values of single particle observables, the next logical step is to look at correlation functions. We therefore calculated the static current-current correlator $\langle\mathrm{GS}|\delta j(x) \delta j(y)| \mathrm{GS}\rangle$ to second order in the impurity tunneling amplitudes $\gamma_{\alpha \beta}$. The zero-order result, which is also the full answer in the case of a static impurity) is (cf. Eq.4.6)

$$
c_{0}(x, y)=-\frac{1}{(2 \pi)^{2}(x-y)^{2}} .
$$

We obtained an expression for the second order correction $c_{2}(x, y)$ to this result (Eq.4.21) that is valid for all $x$ and $y$. It is zero for $x$ and $y$ both to the left or both to the right of the scattering region. Taking $x$ and $y$ on different sides of the scattering region, and the distance 
$|x-y|$ large, we obtained the compact formula

$$
c_{2}(x, y) \simeq-\frac{1}{\pi|x-y|^{3}} \sum_{\alpha}\left(\Delta_{\alpha \lambda}\right)^{2} \partial_{\omega_{\alpha \lambda}} n_{\alpha}\left(\omega_{\alpha \lambda}\right) .
$$

Thus, current-current correlations induced by the impurity show a slow (power-law) decay as a function of distance. These correlations are sub-leading: at large distances, correlations that are present also in the absence of the impurity decay more slowly (second vs. third inverse power of distance). However, the impurity-induced correlations can be detected by varying the impurity parameters, as this leaves the leading order correlations unaffected. Due to the appearance of $n_{\alpha}$ in Eq.6.4 the impurity induced correlations have a power law energy dependence $\sim\left(\omega_{\alpha \lambda}\right)^{\Delta_{\alpha \lambda}^{2}-3}$. Thus correlations are also sensitive to the Fermi edge singularity. The divergence at the Fermi edge threshold for $\Delta_{\alpha \lambda}^{2}<3$ again signals the breakdown of perturbation theory in $\gamma_{\alpha \lambda}$. Understanding correlations in the regime $\left(\Delta_{\alpha \lambda}\right)^{2}<3$ and $\omega_{\alpha \lambda} \rightarrow 0$ is an open problem we are currently working on. In the regime where the Fermi edge singularity result for $n_{\alpha}$ (Eq.6.1) is valid, the correction $c_{2}(x, y)$ is positive. We have argued that $n_{\alpha}$ is always a decreasing function of $\omega_{\alpha \lambda}$. Thus we always expect a positive correction $c_{2}(x, y)$.

Outside the scattering region, the current-current correlator is simply related to the pair correlation function $g(x, y)=\langle\mathrm{GS}|\rho(x) \rho(y)| \mathrm{GS}\rangle$ as

$$
g(x, y)=\bar{\rho}^{2}-\langle\mathrm{GS}|\delta j(x) \delta j(y)| \mathrm{GS}\rangle .
$$

The pair correlation function measures the likelihood to find an electron at $x$ together with another electron at $y$. The positivity of $c_{2}(x, y)$ implies that given an electron at $x$, the likelihood of finding another one at $y$ in the presence of the impurity increases by an amount proportional to $1 /|x-y|^{3}$, for $x$ and $y$ on opposite sides of the scattering region, compared to when the impurity is absent. It is important to note that the increase occurs outside the scattering region, where the average density of electrons is unaffected by the impurity.

\section{Appendix A: The relation between impurity transition rates and ground state occupation probabilities}

In this appendix we derive a relation (Eq.2.12) between the ground state occupation probability $n_{\alpha}$ and the transition rate $W_{\alpha \lambda}$ of the impurity. The starting point is the perturbation expansion of Eqs.4.7 and 4.8 for the ground state. From these equations follow that, to second order in the $\gamma$ 's, $n_{\alpha}$, with $\alpha \neq \lambda$, is given by

$$
n_{\alpha}=\lim _{\eta \rightarrow 0^{+}}\left|\gamma_{\alpha \lambda}\right|^{2} \int_{-\infty}^{0} d t \int_{-\infty}^{0} d t^{\prime} e^{\eta\left(t+t^{\prime}\right)} P_{\alpha \lambda}\left(t-t^{\prime}\right) .
$$

By changing integration variables to $T=\left(t+t^{\prime}\right) / 2$ and $\tau=t-t^{\prime}$, performing the $T$-integral we then obtain

$$
n_{\alpha}=\lim _{\eta \rightarrow 0^{+}} \frac{\left|\gamma_{\alpha \lambda}\right|^{2}}{2 \eta} \int_{-\infty}^{\infty} d \tau e^{-\eta|\tau|} P_{\alpha \lambda}(\tau) .
$$

Substituting for $\left|\gamma_{\alpha \lambda}\right|^{2} P_{\alpha \lambda}(t)$ from (Eq.4.18) allows us to perform the $\tau$-integral. Taking the $\eta \rightarrow 0^{+}$limit, we obtain

$$
n_{\alpha}=\int_{-\infty}^{0} \frac{d \omega}{2 \pi} \frac{W_{\alpha \lambda}(\omega)}{\left(\omega_{\alpha \lambda}-\omega\right)^{2}}
$$

\section{Appendix B: Measurability of the current-current correlator}

We present here what we find to be conceptually the simplest scheme to measure the current-current correlator. Other schemes may be more general or more practical. The Hamiltonian of Eq.2.2 describes right-moving electrons propagating along the real line. A straightforward realization of such a conductor is a quantum Hall edge state. However, another realization is a semiinfinite wire running from $x=-\infty$ to $x=0$, containing both left- and right moving electrons. In this realization, the fermion creation operators $\psi^{\dagger}(x)$ and $\psi^{\dagger}(-x)$ both create an electron at a position $-|x|$, while the sign of $x$ determines whether a left-mover (positive sign) or a right-mover (negative sign) is created. The physically measurable current at $-|x|$ corresponds to the operator

$$
J(-|x|)=j(-|x|)-j(|x|),
$$

where $j(x)$ is the current operator defined in Eq.3.1. In other words, the physically measured current equals the current of right-movers minus the current of left-movers.

In spectral representation, we write $J(-|x|)$ as

$$
J(-|x|)=\sum_{m} J_{m}|m\rangle\langle m|,
$$

where $J_{m}$ and $|m\rangle$ are the many-body eigenvalues and eigenstates of $J(-|x|)$.

By performing current measurements at $-|x|$ on a sufficient number of identical systems prepared in the ground state, the probability density

$$
P(J) \equiv \sum_{m} \delta\left(J_{m}-J\right)|\langle G S \mid m\rangle|^{2},
$$

for an outcome $J$ can approximately be determined. Given $P(J)$, its second moment

$$
M_{2}(-|x|) \equiv \int_{-\infty}^{\infty} d J J^{2} P(J)
$$

can be extracted. From the definitions of $J(-|x|)$ and $P(J)$ follows that

$$
\begin{aligned}
M_{2}(-|x|)= & \left\langle j(|x|)^{2}\right\rangle+\left\langle j(-|x|)^{2}\right\rangle \\
& -\langle j(-|x|) j(|x|)\rangle-\langle j(|x|) j(-|x|)\rangle .
\end{aligned}
$$


In the above expression, expectation values are with respect to the ground state.

The expectation value $\left\langle j(x)^{2}\right\rangle$ turns out to be positionindependent for $x$ outside the range of the impurity interaction, i.e. $x \notin l$. The proof is as follows:

$$
\begin{aligned}
\partial_{x}\left\langle j(x)^{2}\right\rangle & =\left\langle\partial_{x} j(x) j(x)\right\rangle+\left\langle j(x) \partial_{x} j(x)\right\rangle \\
& =i\langle[\rho(x), H] j(x)\rangle+i\langle j(x)[\rho(x), H]\rangle \\
& =i\langle[j(x), H] j(x)\rangle+i\langle j(x)[j(x), H]\rangle \\
& =0 .
\end{aligned}
$$

In the second line we exploited the continuity equation, while in the third line we used the fact that $\rho(x)=j(x)$ (cf. Eq. 3.1) for $x \notin l$. The last line is obtained by noting that the expectation value is with respect to $|\mathrm{GS}\rangle$ which is an eigenstate of $H$.
Thus, $M_{2}(-|x|)$ can be measured, and is given by

$$
M_{2}(-|x|)=M+\langle j(-|x|) j(|x|)\rangle+\langle j(|x|) j(-|x|)\rangle,
$$

where $M$ is independent of $x$. Our main result (Eq.4.26) then translates into a prediction for the $x$ dependent part of $M_{2}$ :

$$
M_{2}(-|x|)-M=-\frac{1}{4 \pi|x|^{3}} \sum_{\alpha}\left(\Delta_{\alpha \lambda}\right)^{2} \partial_{\omega_{\alpha \lambda}} n_{\alpha}\left(\omega_{\alpha \lambda}\right) .
$$

\section{Acknowledgments}

This research was supported by the National Research Foundation (NRF) of South Africa.
* Electronic address: izaksnyman1@gmail.com

1 A. C. Hewson, The Kondo Problem to Heavy Fermions, (Cambridge University Press, Cambridge, UK, 1992).

2 A. K. Geim, P. C. Main, N. La Scala, Jr., L. Eaves, T. J. Foster, P. H. Beton, J. W. Sakai, F. W. Sheard, M. Henini, G. Hill, and M. A. Pate, Phys. Rev. Lett. 72, 2061, (1994).

3 D. Goldhaber-Gordon, H.Shtrikman, D. Mahalu, D. Abusch-Magder, U. Meirav, and M. A. Kastner, Nature 391, 6663, (1998).

4 S. M. Cronenwett, T. H. Oosterkamp, and L. P. Kouwenhoven, Science 281, 5376, (1998).

5 A. L. Moustakas, Quantum impurities in metals. (Ph.D. Thesis, Harvard, 1996).

${ }^{6}$ K. Ohtaka and Y. Tanabe, Rev. Mod. Phys. 62, 929, (1990).

7 P. W. Anderson, Phys. Rev. Lett. 18, 1049, (1967).

8 Y. Adamov and B. Muzykantskii, Phys. Rev. B 64, 245318, (2001).

9 G. D. Mahan, Phys. Rev. 163, 612, (1967).

10 P. Nozières and C. T. DeDominicis, Phys. Rev. 178, 1097, (1969).

11 K. A. Matveev and A. I. Larkin, Phys. Rev. B 46, 15337, (1992).

12 D. A. Abanin and L.S. Levitov, Phys. Rev. Lett. 93, 126802, (2004).

13 M. Heyl and S. Kehrein, Phys. Rev. B 85, 155413 (2012).

14 B. Muzykantskii, N.d'Ambrumenil, and B. Braunecker, Phys. Rev. Lett. 91, 266602, (2003).

15 N. d'Ambrumenil and B. Muzykantskii, Phys. Rev. B 71, 045326, (2005).

16 D. A. Abanin and L. S. Levitov, Phys. Rev. Lett. 94, 186803, (2005).

17 I. Snyman and Yu. V. Nazarov, Phys. Rev. Lett. 99, 096802, (2007).

18 I. Snyman and Yu. V. Nazarov, Phys. Rev. B 77, 165118, (2008).

19 D. B. Gutman, Y. Gefen, and A. D. Mirlin, Phys. Rev. B 81, 085436, (2010).

20 E. Bettelheim, Y. Kaplan, and P. Wiegmann, J. Phys. A: Math. Theor. 44, 282001, (2011).

21 V. V. Mkhitaryan and M. E. Raikh, Phys Rev. Lett. 106,
197003, (2011).

22 N. Maire, F. Hohls, T. Lüdtke, K. Pierz, and R. J. Haug, Phys. Rev. B 75, 233304 (2007).

23 B. Braunecker, Phys. Rev. B 73, 075122, (2006).

24 E. Bettelheim, Y. Kaplan, and P. Wiegmann, Phys. Rev. Lett. 106, 166804, (2011).

25 A. K. Mitchell, M. Becker, and R. Bulla, Phys. Rev. B 84, 115120 (2011).

26 A. Holzner, I. P. McCulloch, U. Schollwock, J. von Delft, F. Heidrich-Meisner, Phys. Rev B, 80, 205114, (2009).

27 S. Oh and J. Kim, Phys. Rev. B 73, 052407, (2006).

28 A. Sheikhan and I. Snyman, Phys. Rev. B 86, 085122, (2012).

29 J. M. Elzerman, R. Hanson, J. S. Greidanus, L. H. Willems van Beveren, S. De Franceschi, L. M. K. Vandersypen, S. Tarucha, and L. P. Kouwenhoven, Phys. Rev. B 67, 161308R, (2003).

30 J. R. Petta, A. C. Johnson, C. M. Marcus, M. P. Hanson, and A. C. Gossard, Phys. Rev. Lett. 93, 186802 (2004).

31 A. Grishin, I. V. Yurkevich, and I. V. Lerner, Phys. Rev. B 72, 060509, (2005).

32 J. Schwinger, Phys. Rev. Lett. 3, 296, (1959).

33 D. C. Mattis and E. H. Lieb, J. Math. Phys. 6, 304, (1965).

34 F. D. M. Haldane, J. Phys. C: Solid State Phys. 14, 2582, (1981).

35 J. von Delft and H. Schoeller, Ann. Phys. 7, 225, (1998).

36 K. D. Schotte and U. Schotte, Phys. Rev. 182, 479, (1969).

37 A. J. Legget, S. Chakravarty, A. T. Dorsey, M. P. A. Fisher, A. Garg, and W. Zwerger, Rev. Mod. Phys. 59, 1, (1987).

38 Y. V. Nazarov and Ya. M. Blanter, Quantum Transport. Introduction to Nanoscience, (Cambridge University Press, Cambridge, UK, 2009). See Chapter 6.2.4.

39 The stated condition on $\omega_{\alpha \lambda}$ is discussed in detail in Sec. VII B of Re. 37 in the context of the spin-boson model with an Ohmic bath.

40 D. C. Langreth, Phys. Rev. 150, 516, (1966).

41 M. Abramowitz and I. A. Stegun, Handbook of Mathematical Functions with Formulas, Graphs, and Mathematical Tables (Dover, N. Y., 1972). See formulas 5.2.12 and 5.2.34. 